Sonderabdruck

aus ,Zeilschrift für Lebensmittel-Untersuchung und -Forschung“"

131. Band, 6. Heft, 1967, Seite 351-353

J.F. Bergmann, München / Springer-I'erlag, Berlin - Heidclberg - New Yorl:

\title{
Infrarotspektroskopischer Mikronachweis von Calciumoxalat in Ananas-Raphiden*
}

Von

Liselote Peters und I. Sucker

Mitteilung aus dem Max-Planck-Institut für Kulturpflanzenzüchtung Hamburg-Volksdorf**

Mit 3 Textabbildungen

(Eingegangen am 7. Februar 1966)

Es ist bekannt, daß in der Ananas Kristalle auftreten, die in den unterhalb der „Fruchtschale“ liegenden Schichten besonders häufig sind. Abb. 1 zeigt einen Gewebeschnitt mit einer typischen bündelähnlichen Anordnung nadelförmiger Kristalle (Raphiden).

DaB es sich hier um Calciumoxalatkristalle handelt, wurde bisher vorwiegend indirekt nachgewiesen: Calciumoxalat ist ohne Aufbrausen (im Gegensatz zu Calciumcarbonat) löslich in Salzsäure, aber unlöslich in Wasser und Essigsäure; mit Schwefelsäure kann es auf dem Objektträger in Calciumsulfat umgewandelt und in Form von Gipskriställchen unter dem Mikroskop er. kannt werden $(1,2,3)$.

Eine qualitativ einwandfreie Identifizierung wird erreicht durch fraktioniertes Zentrifu-

* Herrn Professor Dr. Franz Schwanryz zum 60. Geburtstag gewidmet.

** Wir danken Herrn stud. rer. nat. Hartmut Beug für seine Mitarbeit.

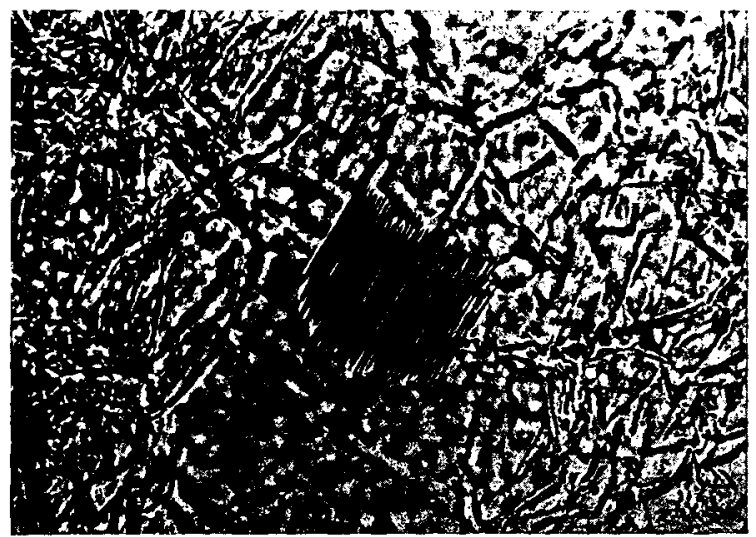

Abb. 1. Mikrophotographie von Kristallnadeln (Raphiden) in Ananasgewebe (Vergrößerung $320 \mathrm{fach}$ ) 
gieren eines in einem Benzol-Bromoform-Gemisch abgestufter Dichte aufgeschwemmten, gepulverten Pflanzengewebes und Aufnahme eines Debye-Scherrer-Röntgendiagrammes des Kristallpulvers $(4,5)$.

Über eine sehr einfache Gewinnung eines Raphidenkonzentrates und den infrarotspektroskopischen Nachweis der Oxalsäure in den Kristallen der Ananas, der auch zur halbquantitativen Bestimmung dienen kann, wird im Folgenden berichtet.

\section{Nachweis}

Isolierung: Von einer Ananas-Frucht wurde ein Teil der "Fruchtschale" und das darunter liegende Gewebe mit einer Schichtdicke von $5 \mathrm{~mm}$ im Mörser zerrieben. Die harten Schalenteile wurden herausgelesen und nach Abspülen mit Wasser entfernt. Das Fruchtfleisch wurde nochmals

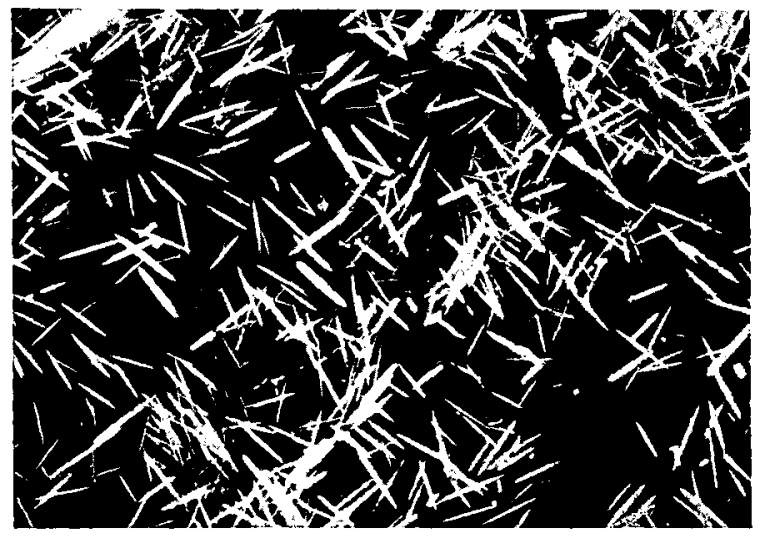

Abb. 2. Mikrophotographie mit polarisiertem Licht: Raphiden-Konzentrat

gemörsert und durch ein grobes Leinentuch filtriert. Die Suspension wurde zentrifugiert, zweimal mit Wasser gewaschen und wieder zentrifugiert. Der erhaltene Bodensatz (,,Raphidenkonzentrat") wurde nach dem Trocknen im Exsiccator mikroskopisch und infrarotspektroskopisch untersucht.

Von dem ,Raphidenkonzentrat" wurde ein Glycerin-GelatineDauerpräparat hergestellt.

Die nadelförmigen Kristalle sind besonders gut in polarisiertem Licht zu erkennen (Abb. 2).

Infrarotspektroskopische Untersuchung: Von dem getrockneten Raphidenkonzentrat wurden etwa $0,3 \mathrm{mg}$ mit $65 \mathrm{mg}$ Kaliumbromid verrieben und zu einer Tablette von $5 \mathrm{~mm}$ Durchmesser gepreßt.

Diese wurde mit Hilfe eines Mikrozusatzes (Beamcondensor und Spezialprobehalter) in einem Beckman-Spektrophotometer IR $4 \mathrm{im}$ Wellenbereich von 1-15 $\mu \mathrm{m}$ gemessen. Das automatisch registrierte Absorptionsspektrum ist in Abb. 3 dargestellt.

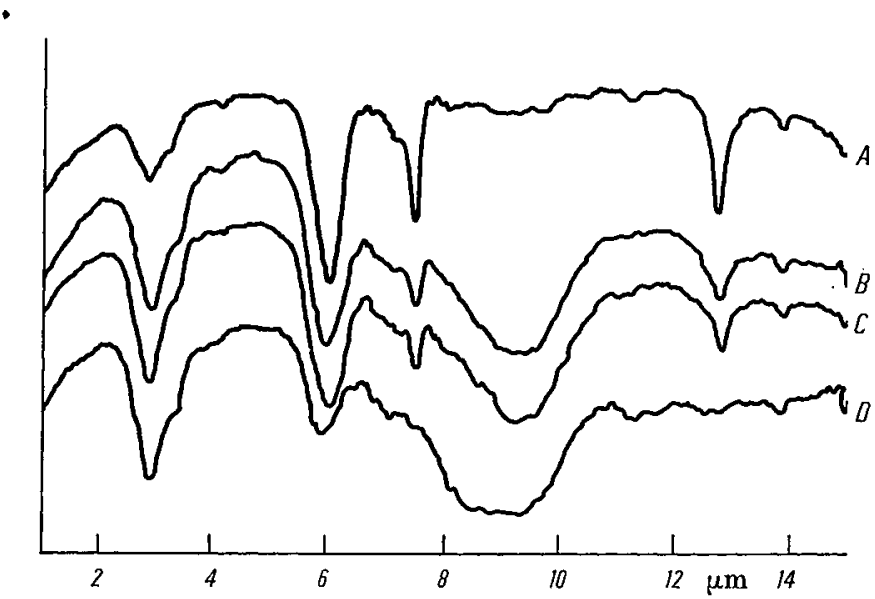

Abb. 3. Infrarotspektren von Ca-oxalat-Präparaten (BECKMAx Spectrophotometer IR4 mit Mikrozusatz), KBr-Preßlinge. A. Calciumoxalat-Monohydrat (Fa. Roth, Karlsruhe); B. Raphidenkonzentrat, unbehandelt; C. Raphidenkonzentrat, mit verdünnter Essigsäure behandelt; D. Raphidenkonzentrat, mit verdünnter Schwefelsäure behandelt. Charakteristische Oxalatbanden: 7,5 und $12,8 \mu \mathrm{m}$ 
Das Kristallkonzentrat wurde zusätzlich entweder je dreimal mit 2 n-Essigsäure oder mit 2 nSchwefelsäure behandelt, zentrifugiert, mit destilliertem Wasser gereinigt und getrocknet. Anschließend wurden Kaliumbromid-Preßlinge hergestellt und die IR-Spektren aufgenommen.

\section{Ergebnis}

Die Abb. 3 A zeigt das Infrarotabsorptionsspektrum eines als Kaliumbromidpreßling gemessenen reinen Calciumoxalat-Monohydrats mit Banden bei 3 und $6 \mu \mathrm{m}(\mathrm{OH}$ Valenz- und $\mathrm{H}_{2} \mathrm{O}$-Deformationsschwingung bzw. Carboxylbande) sowie den beiden für Oxalate typischen Absorptionen bei 7,5 und 12,8 $\mathrm{mm}$. Das Spektrum der Kristallnadeln, des Raphidenkonzentrates (Abb. 3B), stimmt mit reinem Calciumoxalat überein bis auf eine zusätzliche breite Absorption zwischen 9 und $10 \mu \mathrm{m}$, die von der organischen Gerüstsubstanz (Cellulose) herrühren könnte. In Bestätigung der physikalisch-chemischen Eigenschaften des Calciumoxalats liefern die mit Essigsäure behandelten Raphiden (Abb. $3 \mathrm{C}$ ) ein unverändertes Oxalatspektrum, während die Behandlung mit verdünnter Schwefelsäure zur Auflösung des Calciumoxalats führt und im Spektrum (Abb. 3D) daher Oxalatbanden nicht mehr nachweisbar sind. Die untere Nachweisgrenze für Calciumoxalat in einem Oxalat-Phosphat-Gemisch bei normaler infrarotspektroskopischer Meßtechnik mit KBr-Tabletten wurde mit $50 \mu \mathrm{g}$ Calciumoxalat angegeben (6). Der Einsatz des Mikrozusatzes und die Verwendung entsprechend kleiner'Tabletten und geringer Einwaagen bzw. ,,Verdünnung“ der zu messenden Kristalle mit Kaliumbromid gestattet eine Verbesserung der Meßempfindlichkeit um etwa eine Zehnerpotenz; d. h. weniger als $10 \mu \mathrm{g}$ Calciumoxalat lassen sich mit der Mikromethode noch nachweisen.

\section{Zusammenfassung}

Mit Hilfe eines einfachen Verfahrens gelang es, die im Fruchtfleisch der Ananas in Form von Raphiden vorkommenden Kristalle anzureichern und sie im polarisierten Licht mikroskopisch darzustellen. Die infrarotspektroskopische Mikrotechnik liefert den einwandfreien Beweis, daß die nadelförmigen Kristalle aus Calciumoxalat bestehen; außerdem läßt sich diese Methode durch entsprechende Eichmessungen (,,Verdünnungsreihen " analysenreiner Substanzen) für halbquantitative Bestimmungen einsetzen.

\section{Literatur}

1. Brebl, R., u. H. Germ: Praktikum der Pflanzenanatomie. Wien: Springer 1950.

2. STRASSBURGer, E. u. a.: Lehrbuch der Botanik. Stuttgart: Fischer 1962.

3. Walter, H.: Einführung in die Phytologie. Bd. I. Grundlagen des Pflanzenlebens. Stuttgart: Ulmer 1962.

4. v. Philtpsborn, H.: Protoplasma 41, 415 (1952).

5. Paech, K., u. M. V. Tracey : Moderne Methoden der Pflanzenanalyse. Bd. I. Berlin: Springer 1956.

6. SÜCKER, I.: Harnsteinanalyse mit Hilfe physikalisch-chemischer Methoden, speziell Infrarotspektroskopie. Ärztl. Lab. 9, 260 u. 306 (1963). 
Druck: Brühlsche Universitätsdruckerei GieBen 